\title{
PHYTOPLANKTON DIVERSITY IN RELATION TO DIFFERENT WEATHER CONDITIONS IN TWO URBAN MAN MADE LAKES
}

\section{DIVERSIDAD FITOPLANCTON EN RELACIÓN CON DIFERENTES CONDICIONES CLIMÁTICAS EN DOS LAGOS URBANOS DE ORIGEN ANTROPOGÉNICO}

\author{
Munay Abdulqadir Omar ${ }^{1}$, Mohamed Amar Naqqiuddin ${ }^{1}$, Shamarina Shohaimi, Hishamuddin Omar ${ }^{1}$ and \\ Ahmad Ismail ${ }^{*}$ \\ Department of Biology, Faculty of Science, University Putra Malaysia,
} 43400, UPM Serdang, Selangor Darul Ehsan, Malaysia.

${ }^{*}$ ) Correspondent author: aismail@upm.edu.my

\section{Abstract}

Many scientists have reported that global warming have significant impact on phytoplankton community, however, the impact of global warming on phytoplankton communities in suburban made lake is less understood. Therefor the objective of this study are to observe the effect of variable weather conditions on the diversity and succession of phytoplankton in mesotrophic lake (Seri Serdang) and oligotrophic lake (Engineering Faculty Lake). Samples were collected from surface water and species diversity (Shannon Weaver Diversity Index) was calculated. Daily weather and rain fall were recorded. A total of 65 species from five divisions (Chlorophyta, Euglenophyta, Cyanophyta, Bacillariophyta and Dinophyta) and 52 species belonging to six divisions (Chlorophyta, Cyanophyta, Bacillariophyta, Euglenophyta, Cryptophyta \& Charophyta) were recorded from Engineering Faculty Lake and Seri Serdang Lake respectively. Division of Chlorophyta was found most dominant in both lakes during all the weeks (67\%). The most dominant species in Faculty Engineering Lake was Microcystis aeruginosa during all weather conditions. Whereas, the most dominant species in Seri Serdang Lake during all weather conditions were Chlamydomonas reinhardtii, Planktothrix agardhii. The phytoplankton density was low during dry weather conditions for both lakes. The present finding suggested noticeable correlation between weather changes to the alteration of population density of phytoplankton.

Keywords: Trophic index, Chlorophyta, Weather, Lake Ecosystem, Freshwater ecology.

Resumen

Muchos científicos han informado de que el calentamiento global tiene un impacto significativo en la comunidad de fitoplancton, sin embargo, el impacto del calentamiento global en las comunidades de fitoplancton en los suburbios de lago artificial está menos clara. Para ello el objetivo de este estudio es observar el efecto de las condiciones climáticas variables sobre la diversidad y la sucesión de fitoplancton en el lago mesotrófico (Seri Serdang) y el lago oligotrófico (Facultad de Ingeniería Lago). Se recogieron muestras de agua se calculó la superficie y la diversidad de especies (Shannon Índice de Diversidad Weaver). Tiempo actual y la caída de lluvia se registraron. Un total de 65 especies de cinco divisiones (Chlorophyta, Euglenophyta Cianofitas, Bacillariophyta y Dinophyta) y 52 especies pertenecientes a seis divisiones (Chlorophyta, Cianofitas, Bacillariophyta, Euglenophyta, Cryptophyta y Chlorophyta) se registraron a partir de la Facultad de Ingeniería Lago y Seri Serdang Lago, respectivamente. División de Chlorophyta se encontró más dominante en ambos lagos durante todas las semanas (67\%). Las especies más dominantes en la Facultad de Ingeniería Lago fue Microcystis aeruginosa durante todas las condiciones meteorológicas. Mientras que, las especies más dominantes en Seri Serdang lago durante todas las condiciones climáticas eran Chlamydomonas reinhardtii, Planktothrix agardhii. La densidad de fitoplancton fue baja durante el 
tiempo seco para ambos lagos. El presente hallazgo sugiere una correlación notable entre los cambios de clima a la alteración de la densidad de población de fitoplancton.

Palabras clave: índice trófico, Chlorophyta, clima, ecosistema lacustre, ecología de aguas continentales.

Introduction

Phytoplankton diversity and succession in small man-made lakes in Malaysia are largely ignored. Years ago, some of the lakes in Malaysia were studied for phytoplankton and limnological study, however lately there are not many studies have been carried out. As in most part in the world, climate in Malaysia is obviously changing, therefore the changes in water bodies is very likely (Azril et al., 2013). Phytoplankton as biotic component of a lake is directly affected by physical and chemical changes because they are sensitive indicators for variation of conditions in lake ecosystems (Nõges and Tuvikene, 2012). Formation of haze and dust also may reduce light supply to phytoplankton, resulting, low carbon fixation and then low productivity. Excessive amounts of $\mathrm{CO}^{2}$ in the atmosphere are causing acid rain (Dubey, 2013). Rain run-off and untreated sewage flows directly to this water bodies altered the water chemistry and affecting phytoplankton growth (Corcoran. 2010). Atmospheric deposition together with runoff caused changes in lake water chemistry and has profound effects on the aquatic organisms in the lake and one of these organisms is phytoplankton. Although many studies have been conducted to find the potential of phytoplankton in global warming mitigation, most of the studies are done in laboratory (Ramaraj et al., 2014). Hopefully by doing in situ study, may provide better information on phytoplankton composition and succession in each water conditions.

There are about 90 natural and man-made lakes in Malaysia with total area $1,001.821 \mathrm{~km}^{2}$ (Sharip et al., 2014). Phytoplankton species composition were affected by human disturbance in many lakes in Malaysia such as Chini Lake (Mir et al., 2015) and Loagan Bunut (Abdul Rahim et al., 2007). Omar, (2010) Reported using phytoplankton as indicators for water bodies' degradation in Malaysia. The purposes of this work to monitor weekly changes in phytoplankton communities in relation to different weather conditions in oligotrophic lake and mesotrophic lakes, with referring to physical environmental conditions and also no phytoplankton studies have been done in these Chosen lakes for this study.

Materials and Methods

The sampling was done in two urban manmade lakes, Faculty of Engineering (UPM) as oligotrophic lake, and Seri Serdang as Mesotrophic Lake (Figure 1). Sampling stations were located at the edge of both lakes, from the littoral zone. The Faculty of Engineering Lake is located in the middle of the academic building of Engineering Faculty (UPM) at $\left(3^{\circ} 00^{\prime} \mathrm{N}, 101^{\circ} 72^{\prime} \mathrm{E}\right)$. It is used for recreational and storm water storage. The total surface area of the lake is of $24,140 \mathrm{~m}^{2}$ and the maximum depth is about $5 \mathrm{~m}$. Water source is commonly from precipitation and runoff (Adeleke et al., 2014). The lake has one inlet point and one outlet point. The Seri Serdang Lake is located near the housing estate of Sri Serdang at $\left(3^{\circ} 00^{\prime} \mathrm{N}\right.$, $\left.101^{\circ} 71^{\prime} \mathrm{E}\right)$. It is connected to the end of stream connecting Kuyoh River. Smaller stream connecting to Kuyoh River is the tributary of the main Kuyoh River. The total surface area is about $18,000 \mathrm{~m}^{2}$ and the deepest part is about 5 meter depth (Abdillah and Teo .2013). The Lake has four inlet points and one outlet point. 


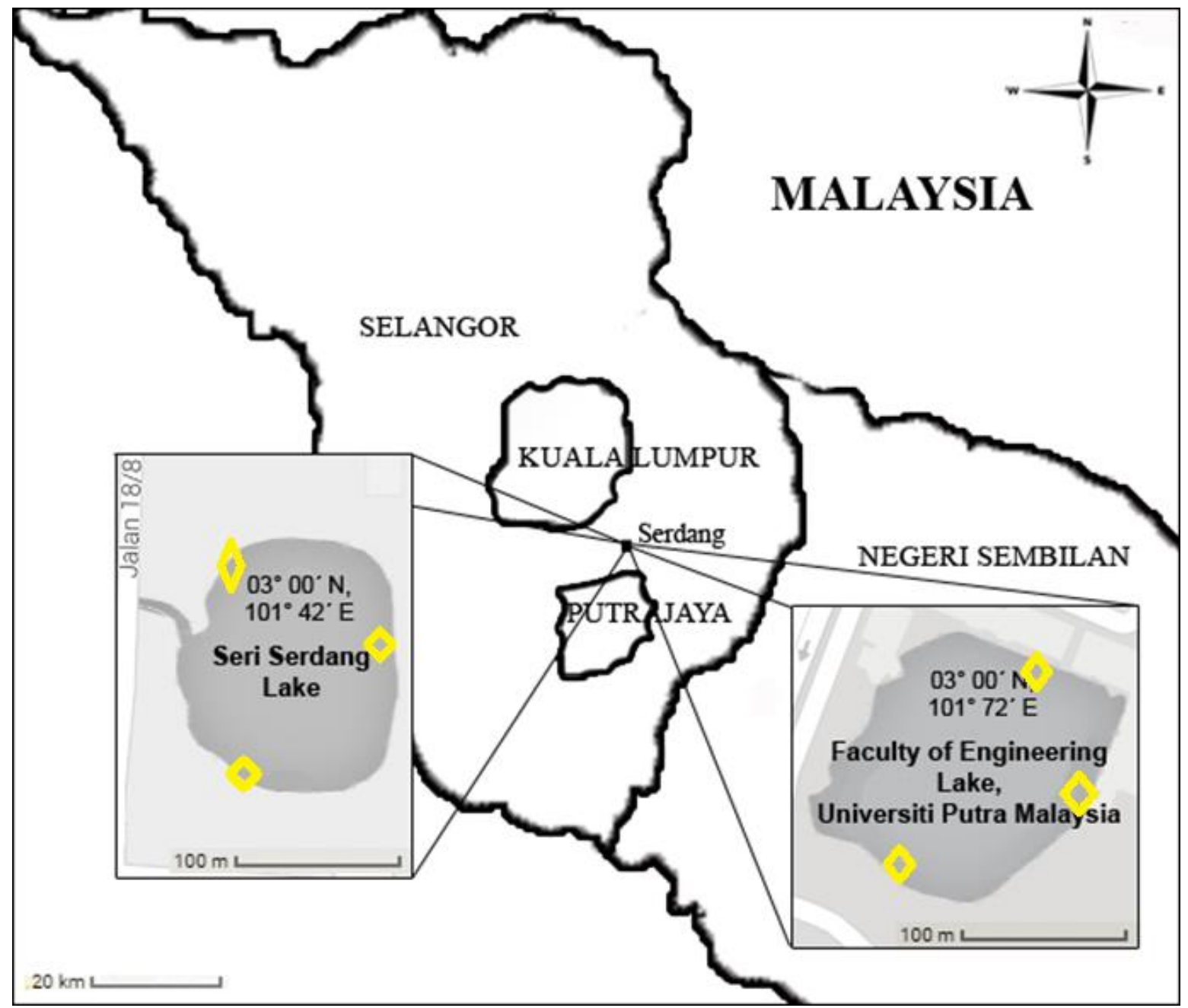

Fig. 1: Summary of google Maps of sampling location, Seri Serdang Lake and Engineering Faculty Lake.

Weekly sampling was done from September 2014 to January 2015 and July of 2015. Light intensity $\left(\mu \mathrm{mol} . \mathrm{m}^{-2} . \mathrm{s}^{-1}\right)$ was determined by using light meter Licor model (L1-250). $\mathrm{pH}$, conductivity, total dissolved solids, salinity, dissolved oxygen and water temperature were taken by Yellow Spring Instrument multi parameter probe model (YSI-556 MPS) from surface water. Water transparency was measured by Secchi disk (20 cm diameter). Triplicate sampling of $500 \mathrm{ml}$ water samples were done for phytoplankton analysis from surface water at $0.5 \mathrm{~m}$ depth by modified collection bottle. The sites and the time of sampling were fixed for every week. The abundance of algae was estimated by the Utermöhl method (Utermohl, 1958). The samples were fixed by adding 10 drops of glutaraldehyde solution (Kumar. 2012). The samples were left undisturbed to allow phytoplankton to settle down for at least 48h (Edler and Elbrächter. 2010). Then 400 $\mathrm{ml}$ were moved gradually from surface of the bottle, then 1drop from the remaining $100 \mathrm{ml}$ were placed into Haemocytometer counting chamber (three times from each sample). Phytoplankton were identified according to a key of freshwater algae (Bellinger and Sigee. 2010) and counted by Olympic light microscope at magnification of 400x. Phytoplankton diversity was calculated by Shannon-Weaver index (Shannon and Weaver. 1949), Simpson index, and Evenness and Species richness (Margalef, 1958). Sorenson's coefficient (Sørensen. 1948) to determine the similarity in phytoplankton diversity between both lakes. Nygaard's index (Nygaard, 1949) was calculated for trophic status. Air temperature (Accuweather.com), light intensity by using light meter Licor model (L1-250), and weather state (rainy, cloudy, sunny, haze) were recorded daily three time a day at $8: 30 \mathrm{am}, 12: 30 \mathrm{pm}$, and $4: 30 \mathrm{pm}$. To determine rainfall, rain gauge was constructed from $5 \mathrm{~L}$ size bottles with fixed funnel at the top and placed beside each lake and the volume of water in the gauge 
was measured after each raining time (Goldstein, 2002). pH by using (METTLER TOLEDO) meter and water volume were determined for the rain water collected.

Calculation formula:

Rain fall gauging $(\mathrm{mm})=$ (Collected water volume) / (funnel surface area/bottle base area)

One-way ANOVA statistical analysis from SPSS version 21 was used to indicate the significant of variance in physical environmental parameters and species diversity indices among different sampling periods. Spearman Correlation Coefficient was determined to correlate temperature $\left({ }^{\circ} \mathrm{C}\right)$ and diversity Index. Canonical Correspondence Analysis (CCA) was conducted to detect species distribution related to physical environmental parameters and different weather conditions.

\section{Results}

Based on weather recording of six months of sampling, the weather conditions can be classified into three weather conditions, mix (3 September to 15 October), wet (22 October to 10 December 2015) and dry (17 December to 22 January and from 2 to 9 of June 2015) as listed in (Table 1).

Table 1. Average of air temperature and light intensity, and the weather status for three different weather conditions in the study area.

\begin{tabular}{lrrl}
\hline $\begin{array}{l}\text { Weather } \\
\text { category }\end{array}$ & $\begin{array}{l}\text { Air temperature } \\
\left.{ }^{\circ} \mathrm{C}\right)\end{array}$ & Light intensity $\left(\mu \mathrm{molm}^{-2} \mathrm{~s}^{-1}\right)$ & Weather status \\
\hline Mix & $29.0 \pm 0.28$ & $400.5 \pm 38.0$ & $\begin{array}{l}\text { Mostly cloudy, haze, rains two or } \\
\text { three times a week }\end{array}$ \\
Wet & $28.8 \pm 0.28$ & $498.7 \pm 36.6$ & $\begin{array}{l}\text { Cloud cover, heavy rain most the } \\
\text { week }\end{array}$ \\
Dry & $28.6 \pm 0.27$ & $700.2 \pm 32.3$ & Sunny, rain once a week or not rainy \\
\hline
\end{tabular}

There is no statistically significant difference in $\mathrm{pH}$ and nitrate content of rain water in Engineering lake $(p>0.05)$, but there is a significant difference in rainfall collected $(p<0.05)$. There is no statistically significant difference $(p>0.05)$ in $\mathrm{pH}$ and nitrate content in rain water gauged beside Seri Serdang Lake. There is a significant difference in rain water volume collected between the different weather conditions ( $p$ $<0.05$ ) as shown in (Table 2).

Table 2. Rain water parameters in different weather conditions (mix, wet and dry).

\begin{tabular}{lcccccc}
\hline Parameter & \multicolumn{3}{c}{ Engineering Faculty Lake } & \multicolumn{3}{c}{ Seri Serdang Lake } \\
\cline { 2 - 7 } & Mix & Wet & Dry & Mix & Wet & Dry \\
Volume of water gauged & 158.2 & 741.6 & 40.7 & 266.1 & 750.5 & 48.60 \\
$(\mathrm{~mm})$ & \pm 99.9 & \pm 190.3 & \pm 12.4 & \pm 139 & \pm 186.6 & \pm 16.4 \\
$\mathrm{pH}$ values & $5.5 \pm 0.11$ & $5.2 \pm 0.18$ & $5.3 \pm 0.22$ & $5.6 \pm 0.15$ & $5.3 \pm 0.22$ & $5.3 \pm 0.22$ \\
\hline
\end{tabular}


The physical parameters of lake water and their values that affect the succession of phytoplankton in the both lakes are presented in (Table 3). There was a significant difference in water temperature, salinity and secchi depth ( $p<0.05$ ), while there was no significant differences in the other parameters in Engineering Faculty Lake. In Seri Serdang Lake there was a significant difference in water temperature, conductivity, total dissolved solids and salinity $(p<0.05)$, while there was no significant differences in the other parameters.

Table 3. The average of physical environmental parameters in surface water layers in the both lakes $(n=7$ (mix), $n=7$ (dry) and $n=8$ (wet)).

\begin{tabular}{|c|c|c|c|c|c|c|}
\hline \multirow[t]{2}{*}{ Parameters } & \multicolumn{3}{|c|}{ Engineering Faculty Lake } & \multicolumn{3}{|c|}{ Seri Serdang Lake } \\
\hline & Mix & Wet & Dry & Mix & Wet & Dry \\
\hline \multirow[t]{2}{*}{$\mathrm{pH}$} & 7.23 & 7.19 & 7.02 & 7.33 & 6.95 & 7.06 \\
\hline & \pm 0.20 & \pm 0.17 & \pm 0.14 & \pm 0.20 & \pm 0.21 & \pm 0.12 \\
\hline \multirow[t]{2}{*}{ Secchi photic depth (m) } & 0.304 & 0.423 & 0.291 & 0.214 & 0.248 & 0.199 \\
\hline & \pm 0.005 & \pm 0.02 & \pm 0.017 & \pm 0.02 & \pm 0.011 & \pm 0.02 \\
\hline \multirow{3}{*}{$\begin{array}{l}\text { Light intensity } \\
\left(\mu \mathrm{molm}{ }^{-2} \mathrm{~s}^{-1}\right)\end{array}$} & 731.8 & 768.0 & 752.2 & 865.4 & 761.5 & 529.7 \\
\hline & \pm 148.2 & & & +1461 & +7485 & \\
\hline & & & & & & \\
\hline \multirow{2}{*}{$\begin{array}{l}\text { Surface Water Temperature } \\
(\stackrel{\circ}{ }) \text { ) }\end{array}$} & 29.12 & 27.98 & $29.75 \pm 0.14$ & 29.57 & 28.7 & 30.03 \\
\hline & \pm 0.20 & \pm 0.24 & & \pm 0.60 & \pm 0.19 & \pm 0.14 \\
\hline \multirow[t]{2}{*}{ Conductivity $\left(\mathrm{mS} \mathrm{cm}^{-1}\right)$} & 0.118 & 0.097 & 0.236 & 0.484 & 0.350 & 0.408 \\
\hline & \pm 0.001 & \pm 0.003 & \pm 0.120 & \pm 0.019 & \pm 0.020 & \pm 0.000 \\
\hline Total dissolved solids & 76.00 & 66.00 & 68.00 & 284.0 & 218.0 & 257.0 \\
\hline$\left(\mathrm{mg} \mathrm{L}^{-1}\right)$ & \pm 0.007 & \pm 0.006 & \pm 0.001 & \pm 0.011 & \pm 0.01 & \pm 0.01 \\
\hline Dissolved Oxygen & 3.19 & 6.78 & 6.39 & 3.76 & 4.67 & 4.93 \\
\hline$\left(\mathrm{mg} \mathrm{L}^{-1}\right)$ & \pm 0.44 & \pm 2.01 & \pm 1.76 & \pm 0.61 & \pm 1.22 & \pm 2.21 \\
\hline \multirow[t]{2}{*}{ Salinity (ppt) } & 0.05 & 0.042 & 0.047 & 0.20 & 0.15 & 0.19 \\
\hline & \pm 0.00 & \pm 0.00 & \pm 0.001 & \pm 0.008 & \pm 0.008 & \pm 0.01 \\
\hline
\end{tabular}

During 22 weeks, 65 species following to 38 genus and 5 divisions were recorded from Engineering Faculty Lake (UPM) as in (Table 4). The most diverse group was Chlorophyta (67.6\%), followed by Dinophyta (13.8\%), Cyanophyta (7.6\%), Bacillariophyta (7.6\%), and Euglenophyta (3.07\%). Fourteen species were dominant during the mix weather conditions, Chlorophyta formed (71\%) of the dominance. While 10 species were dominant during the wet weather conditions, Chlorophyta formed (70\%) of the dominance. During dry weather conditions, 12 species were dominant and Chlorophyta had the big chance by (91/\%) of the dominance. Fifty two species following to 38 genus and 6 divisions were recorded from Seri Serdang Lake 
(Table 5). The most diverse group was Chlorophyta (67.3\%), followed by Euglenophyta (11.5\%) and Cyanophyta (11.5\%), Bacillarophyta (5.7\%), then the rare groups, Charophyta (1.9\%) and Chrysophyta $(1.9 \%)$. Twelve species were dominant during the mix weather conditions, Chlorophyta reached $(41 \%)$ of the dominance. 18 species were dominant under wet weather conditions, Chlorophyta comprised (66.6\%) of the dominance. During dry weather conditions, 16 species were dominant and Chlorophyta were the most dominant group by $(75 \%)$.

Phytoplankton diversity Indices of Engineering Lake and Seri Serdang Lake were presented in (Table 6). The lowest species diversity was observed in both lakes in dry weather condition, while the highest was in mix weather conditions in Engineering Lake, and in wet weather conditions for Seri Serdang Lake. There is a significant difference $(p<0.05)$ in Shannon index and Simpson index in Engineering lake, but there is no significant difference in species richness. While there is no significant difference in diversity indices in Seri Serdang lake.
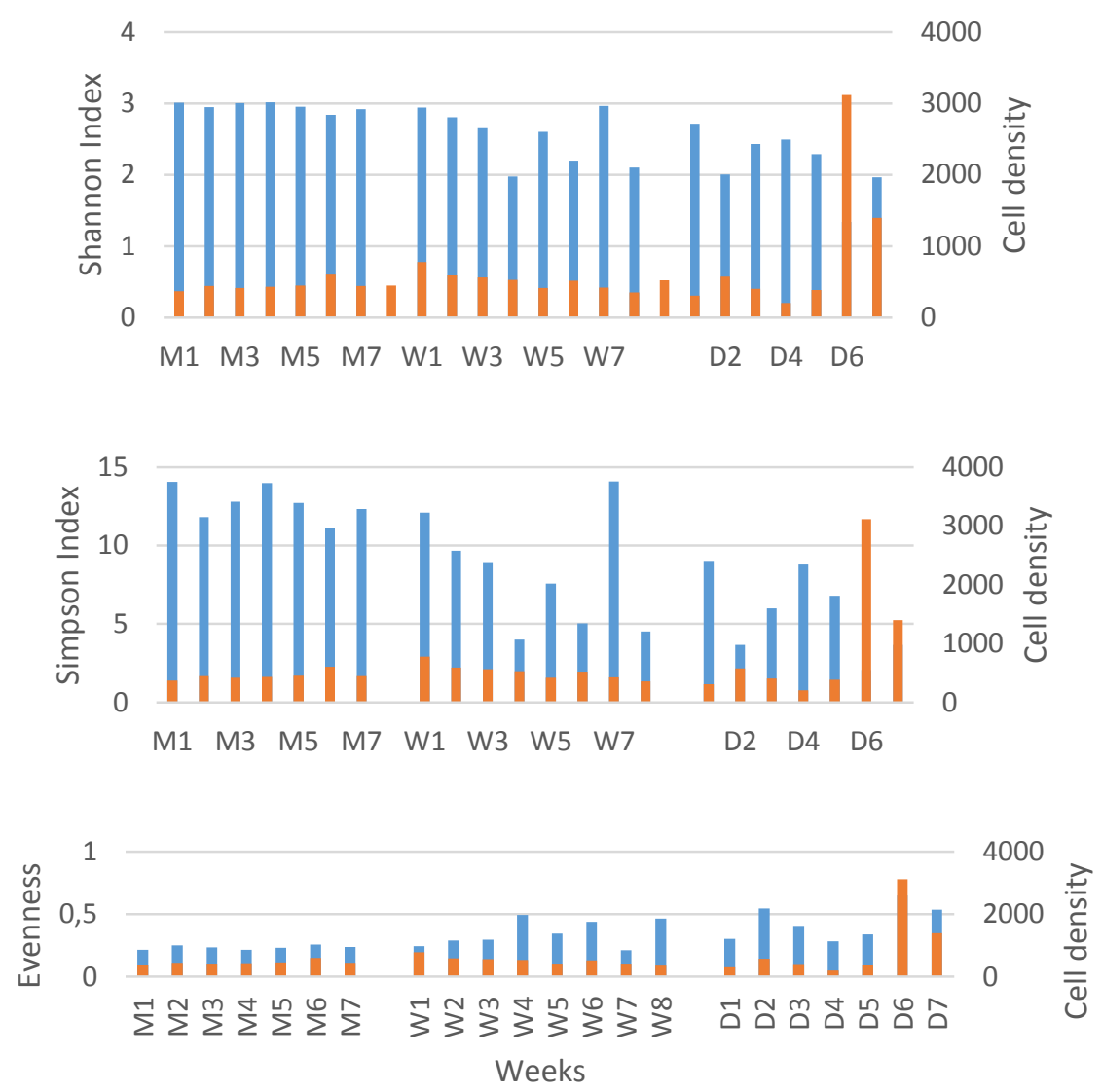

- Shannon Index/ Simpson Index/ Evenness $\quad$ Cell density

Fig. 2. Seasonal variation of species diversity indices in Faculty of Engineering Lake. 
Sustainability, Agri, Food and Environmental Research 4(1), 2016: 1-21

ISSN: 0719-3726
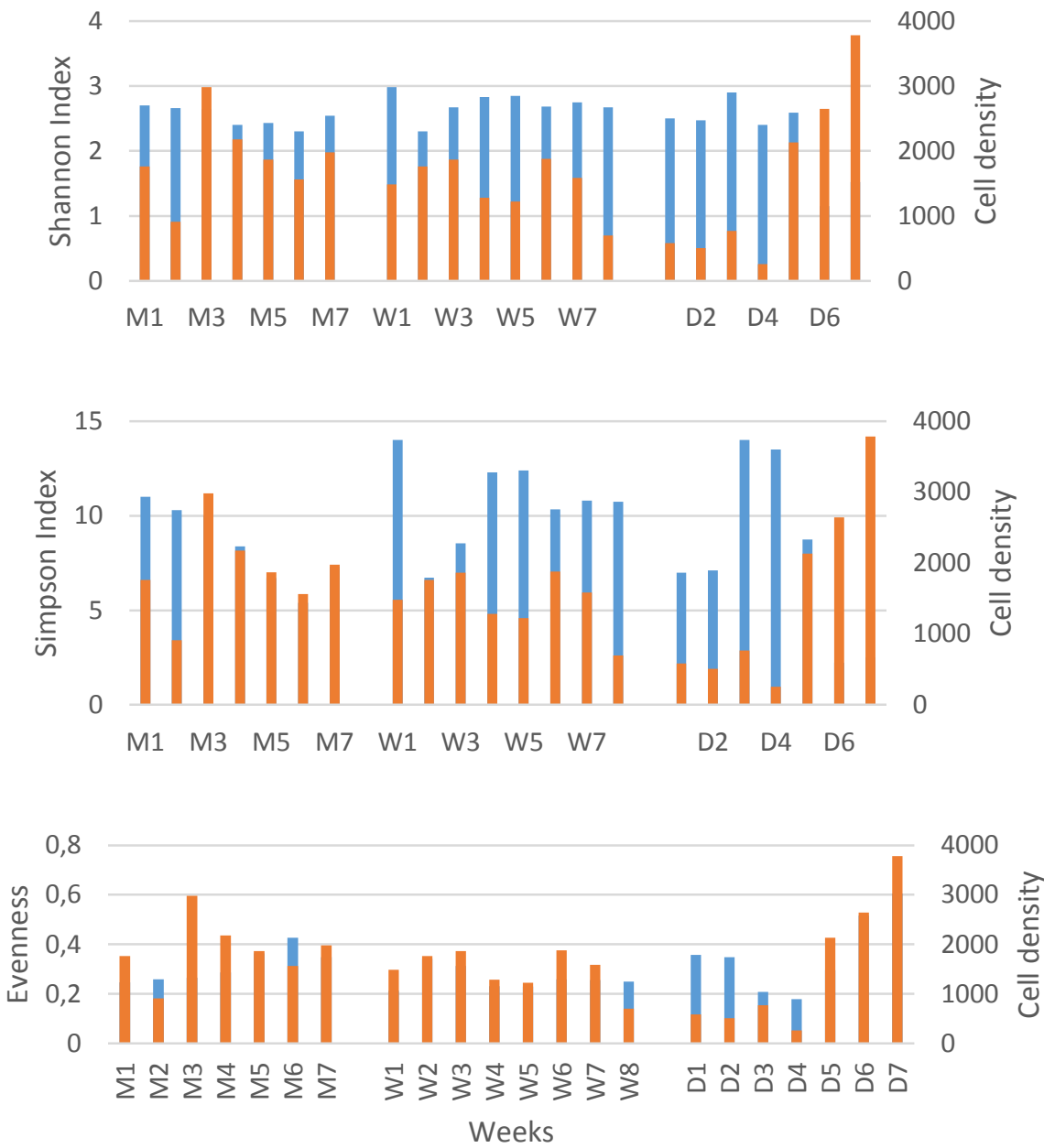

- Shannon Index/Simpson Index/ Evenness $\quad$ Cell Density

Fig. 3. Seasonal variation of species diversity indices in Seri Serdang Lake.

The similarity species composition between the two lakes in different weather conditions:

In total thirty four species were common in the both lakes, but the similarity in species composition degraded slightly during different weather conditions, it was 13,11 , and 9 combined species during mix, wet and dry weather conditions respectively. Chlamydomonas reinhardtii, Dictyosphaerium pulchellum, Kirchneriella obesa, Monoraphidium griffithii, Oocystis borgei, Microcystis aeruginosa, were found in the two lakes during all weather conditions, while Scenedesmus obliquus, Scenedesmus bicaudata and Chrococcus targidus were found in the both lakes only during wet weather conditions.

The principal robust species in the two lakes in different weather conditions:

The number of most occurrence species in engineering lake were $(14,11$, and 12$)$ species and in Seri Serdang Lake were $(12,18$, and 16$)$ species during mix, wet and dry weather respectively and they are presented in (Table 7) for Faculty of Engineering Lake and Seri Serdang Lake.

Nygaard's index calculated as ratio of algae groups to desmids, since desmids number in Engineering Faculty Lake were (7, 5 and 5$)$ and in Seri Serdang Lake $(3,2$ and 1$)$ during mix, wet, and dry weather conditions respectively (Table 8 ).

Statistical relationships between the composition of phytoplankton and the physical environmental parameters in the different weather conditions in the surface water in both lakes were presented in (Figure 4 and Figure 5). 
Sustainability, Agri, Food and Environmental Research 4(1), 2016: 1-21

ISSN: 0719-3726

Table 4. List of phytoplankton species categorized in Engineering Faculty Lake.

\begin{tabular}{|c|c|c|c|}
\hline Chlorophyta & M & W & $\mathrm{D}$ \\
\hline Ankistrodesmus fusiformis Corda & $\mathrm{R}$ & $\mathrm{R}$ & $\mathrm{R}$ \\
\hline Actinastrum hantzschii Lagerheim & $\mathrm{R}$ & $\mathrm{R}$ & $\mathrm{R}$ \\
\hline Chlamydomonas reinhardtii P.A.Dangeard & $\mathrm{S}$ & $\mathrm{S}$ & $\mathrm{S}$ \\
\hline Cosmarium undulatum Corda ex Ralfs & $\mathrm{F}$ & $\mathrm{R}$ & $\mathrm{F}$ \\
\hline Crucigenia quadrata Morren & $\mathrm{F}$ & $\mathrm{S}$ & $\mathrm{S}$ \\
\hline Crucigenia tetrapedia Kuntze & $\mathrm{F}$ & $\mathrm{F}$ & $\mathrm{R}$ \\
\hline Crucigenia crucifera O.Kuntze & $\mathrm{F}$ & $\mathrm{F}$ & $\mathrm{S}$ \\
\hline Dictyosphaerium pulchellum H.C.Wood & $S$ & $\mathrm{~F}$ & $\mathrm{~S}$ \\
\hline Euastrum biverrucosum A.A.Gontcharov \& M.M.Watanabe & $\mathrm{R}$ & $\mathrm{R}$ & $\mathrm{R}$ \\
\hline Eudorina elegans Ehrenberg & $\mathrm{F}$ & $\mathrm{F}$ & $\mathrm{R}$ \\
\hline Golenkinia radiata Chodat & $\mathrm{R}$ & $\mathrm{F}$ & $\mathrm{F}$ \\
\hline Gonium pectorale O.F.Müller & $\mathrm{F}$ & $\mathrm{S}$ & $\mathrm{F}$ \\
\hline Kirchneriella obesa West \& G.S.West & $\mathrm{S}$ & $\mathrm{S}$ & $\mathrm{F}$ \\
\hline Micractinium pusillum Fresenius & $\mathrm{F}$ & $\mathrm{R}$ & $\mathrm{R}$ \\
\hline Micractinium radiatum Wille & $\mathrm{F}$ & $\mathrm{R}$ & $\mathrm{R}$ \\
\hline Monoraphidium griffithii Komárková-Legnerová & $\mathrm{F}$ & $\mathrm{F}$ & $\mathrm{F}$ \\
\hline Monoraphidium arcuatum Hindák & $\mathrm{F}$ & $\mathrm{F}$ & $\mathrm{R}$ \\
\hline Monoraphidium contortum Komárková-Legnerová & $\mathrm{R}$ & $\mathrm{R}$ & $\mathrm{F}$ \\
\hline Micrasterias furcata C.Agardh ex Ralfs & $\mathrm{F}$ & $\mathrm{R}$ & $\mathrm{R}$ \\
\hline Pandorina morum Bory & $\mathrm{F}$ & $\mathrm{F}$ & $\mathrm{R}$ \\
\hline Pediastrum boryanum Meneghini & $\mathrm{F}$ & $\mathrm{F}$ & $\mathrm{R}$ \\
\hline Pediastrum duplex Meyen & $\mathrm{F}$ & $\mathrm{F}$ & $\mathrm{F}$ \\
\hline Pediastrum simplex Meyen & $\mathrm{F}$ & $\mathrm{F}$ & $\mathrm{F}$ \\
\hline Scenedesmus quadricauda Brébisson & $\mathrm{S}$ & $\mathrm{S}$ & $\mathrm{S}$ \\
\hline Scenedesmus acuminatus Chodat & $\mathrm{F}$ & $\mathrm{F}$ & $\mathrm{F}$ \\
\hline Scenedesmus armatus R.Chodat & $\mathrm{F}$ & $\mathrm{F}$ & $\mathrm{F}$ \\
\hline Scenedesmus bicaudatus Dedusenko & $\mathrm{S}$ & $\mathrm{S}$ & $\mathrm{S}$ \\
\hline Scenedesmus obtusus Meyen & $\mathrm{F}$ & $\mathrm{R}$ & $\mathrm{R}$ \\
\hline Scenedesmus opalensis P.G.Richter & $\mathrm{F}$ & $\mathrm{R}$ & $\mathrm{R}$ \\
\hline Scenedesmus obliquus Kützing & $\mathrm{S}$ & $\mathrm{S}$ & $\mathrm{S}$ \\
\hline Scenedesmus dimorphus Kützing & $\mathrm{R}$ & $\mathrm{R}$ & $\mathrm{R}$ \\
\hline Scenedesmus abundans Chodat & $\mathrm{R}$ & $\mathrm{F}$ & $\mathrm{S}$ \\
\hline Scenedesmus ellipticus Corda & $\mathrm{S}$ & $\mathrm{S}$ & $\mathrm{F}$ \\
\hline Staurastrum muticum Brébisson ex Ralfs & $\mathrm{F}$ & $\mathrm{F}$ & $\mathrm{R}$ \\
\hline Staurodesmus triangularis Teiling & $\mathrm{R}$ & $\mathrm{R}$ & $\mathrm{F}$ \\
\hline Staurodesmus incus Teiling & $\mathrm{F}$ & $\mathrm{R}$ & $\mathrm{R}$ \\
\hline Staurodesmus glaber Teiling & $\mathrm{F}$ & $\mathrm{R}$ & $\mathrm{F}$ \\
\hline Tetraëdron caudatum Hansgirg & $\mathrm{F}$ & $\mathrm{F}$ & $\mathrm{R}$ \\
\hline Tetraëdron minimum Hansgirg & $\mathrm{F}$ & $\mathrm{F}$ & $\mathrm{F}$ \\
\hline Tetraëdron triangulare Korshikov & $\mathrm{F}$ & $\mathrm{F}$ & $\mathrm{R}$ \\
\hline
\end{tabular}


Sustainability, Agri, Food and Environmental Research 4(1), 2016: 1-21

ISSN: 0719-3726

Table 4, continuation.

Lagerheimia ciliata Chodat

Oocystis borgei J.W.Snow

Ulothrix aequalis Kützing

Westella botryoides De Wildeman

Cyanophyta

Chroococcus minimus Lemmermann

Chroococcus turgidus Nägeli

Microcystis aeruginosa Kützing

Microcystis wesenbergii Komárek ex Komárek

Merismopedia punctata Meyen

Bacillariophyta

Aulacoseira granulata Simonsen

Cymbella lanceolata Kirchner

Cyclotella meneghiniana Kützing

Navicula rhynchocephata

Nitzschia amphibia Grunow

Dinophyta

Peridinium umbonatum Stein

Peridinium cinctum Ehrenberg

Protoperidinium thulesense Balech

Prorocentrum micans Ehrenberg

Gymnodinium helveticum Penard

Gymnodinium aureolum Gert Hansen

Dinophysis acuta Ehrenberg

Gyrodinium uncatenum Hulburt

Glenodinium danicum Paulsen

$\mathrm{R}$

$\mathrm{S}$

$\mathrm{F}$

$\mathrm{F}$

$\mathrm{F}$

$\mathrm{F}$

$\mathrm{R}$

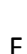

$\mathrm{F}$

$\mathrm{S}$

$\mathrm{S}$

$\mathrm{S}$

$\mathrm{S}$

$\mathrm{R}$

Euglenophyta

Euglena viridis Ehrenberg

Trachelomonas hispida F.Stein

\begin{tabular}{|c|c|c|}
\hline$F$ & $F$ & $F$ \\
\hline $\mathrm{R}$ & $F$ & $\mathrm{R}$ \\
\hline $\mathrm{R}$ & $F$ & $F$ \\
\hline $\mathrm{F}$ & $F$ & $F$ \\
\hline$F$ & $\mathrm{R}$ & $\mathrm{R}$ \\
\hline $\mathrm{F}$ & $F$ & $F$ \\
\hline$F$ & $\mathrm{R}$ & $\mathrm{R}$ \\
\hline $\mathrm{R}$ & $\mathrm{R}$ & $\mathrm{R}$ \\
\hline$F$ & $\mathrm{R}$ & $\mathrm{R}$ \\
\hline$F$ & $F$ & $F$ \\
\hline$F$ & $\mathrm{R}$ & $F$ \\
\hline$F$ & $F$ & $\mathrm{R}$ \\
\hline$F$ & $\mathrm{R}$ & $F$ \\
\hline$F$ & $\mathrm{~F}$ & $F$ \\
\hline$F$ & $F$ & $F$ \\
\hline$F$ & $S$ & $F$ \\
\hline
\end{tabular}

M: Mix Weather

W: Wet Weather

$\mathrm{R}=$ rare occurrence, $<1000$ cells $/ \mathrm{ml}$

D: Dry Weather

$\mathrm{F}=$ frequent occurrence, $1000-9999$ cells $/ \mathrm{ml}$

$\mathrm{S}=$ subdominant, $10000-99999$ cells $/ \mathrm{ml}$

$\mathrm{D}=$ dominant, $>100000$ cells $/ \mathrm{ml}$ [29] 
Sustainability, Agri, Food and Environmental Research 4(1), 2016: 1-21

ISSN: 0719-3726

Table 5. List of phytoplankton species categorized in Seri Serdang Lake.

\begin{tabular}{|c|c|c|c|}
\hline Chlorophyta & $\mathrm{M}$ & W & $\bar{D}$ \\
\hline Actinastrum hontzchii Lagerheim & $\mathrm{F}$ & $\mathrm{S}$ & $\mathrm{s}$ \\
\hline Ankistrodesmus fusiformis Corda & $\mathrm{s}$ & $\mathrm{F}$ & $\mathrm{F}$ \\
\hline Chlamydomonas reinhardatii P.A.Dangeard** & $\mathrm{D}$ & $\mathrm{D}$ & $\mathrm{D}$ \\
\hline Chlorella vulgaris Beyerinck & D & $\mathrm{s}$ & $\mathrm{s}$ \\
\hline Chlorogonium elongatum Francé & $\mathrm{S}$ & $\mathrm{D}$ & $\mathrm{s}$ \\
\hline Coelastrum microporum Nägeli & $\mathrm{F}$ & $\mathrm{F}$ & $\mathrm{F}$ \\
\hline Dictyosphaerium pulchellum H.C.Wood & $\mathrm{S}$ & $\mathrm{s}$ & $\mathrm{s}$ \\
\hline Eudorina unicocca G.M.Smith & $\mathrm{s}$ & $\mathrm{s}$ & $\mathrm{F}$ \\
\hline Gloeocapsa punctata Nägeli & $\mathrm{R}$ & $\mathrm{F}$ & $\mathrm{F}$ \\
\hline Golenkinia radiata Chodat & $\mathrm{F}$ & $\mathrm{s}$ & $\mathrm{s}$ \\
\hline Gonium pectorale O.F.Müller & $\mathrm{F}$ & $\mathrm{F}$ & $\mathrm{F}$ \\
\hline Haematococcus pluvialis Flotow & $\mathrm{F}$ & D & $\mathrm{s}$ \\
\hline Kirchneriella obesa West \& G.S.West & $\mathrm{S}$ & $\mathrm{s}$ & $\mathrm{F}$ \\
\hline Lagerheimia ciliate Chodat & $\mathrm{F}$ & $\mathrm{F}$ & $\mathrm{F}$ \\
\hline Micractinium quadrisetum G.M.Smith & $\mathrm{S}$ & $\mathrm{F}$ & $\mathrm{F}$ \\
\hline Micractinium pusillum Fresenius & $\mathrm{S}$ & $\mathrm{s}$ & $\mathrm{s}$ \\
\hline Micractinium bornhemiense Korshikov & $\mathrm{R}$ & $\mathrm{R}$ & $\mathrm{R}$ \\
\hline Monoraphidium griffithii Komárková-Legnerová & $\mathrm{S}$ & $\mathrm{s}$ & $\mathrm{S}$ \\
\hline Monoraphidium arcuatum Hindák & $\mathrm{S}$ & $\mathrm{s}$ & $\mathrm{F}$ \\
\hline Monoraphidium contortum Komárková-Legnerová & $\mathrm{F}$ & $\mathrm{F}$ & $\mathrm{F}$ \\
\hline Oocystis borgei J.W.Snow & $\mathrm{s}$ & $\mathrm{s}$ & $\mathrm{S}$ \\
\hline Pandorina morum Bory & $\mathrm{D}$ & $\mathrm{s}$ & $\mathrm{F}$ \\
\hline Pteromonas aculeata Lemmermann & $\mathrm{F}$ & $\mathrm{D}$ & $\mathrm{S}$ \\
\hline Scenedesmus quadricauda Brébisson & $\mathrm{F}$ & $\mathrm{s}$ & $\mathrm{F}$ \\
\hline Scenedesmus acuminatus Chodat & $\mathrm{R}$ & $\mathrm{R}$ & $\mathrm{F}$ \\
\hline Scenedesmus armatus R.Chodat & $\mathrm{F}$ & $\mathrm{F}$ & $\mathrm{F}$ \\
\hline Scenedesmus opoliensis P.G.Richter & $\mathrm{F}$ & $\mathrm{F}$ & $\mathrm{F}$ \\
\hline Scenedesmus obliquus Kützing & $\mathrm{F}$ & $\mathrm{F}$ & $\mathrm{R}$ \\
\hline Scenedesmus bicaudatus Dedusenko & $\mathrm{F}$ & $\mathrm{S}$ & $\mathrm{F}$ \\
\hline Scenedesmus dimorphis Kützing & $\mathrm{F}$ & $\mathrm{F}$ & $\mathrm{F}$ \\
\hline Staurastrum punctulatum Brébisson & $\mathrm{F}$ & $\mathrm{F}$ & $\mathrm{R}$ \\
\hline Staurastrum muticum Brébisson ex Ralfs & $\mathrm{R}$ & $\mathrm{F}$ & $\mathrm{R}$ \\
\hline Staurodesmus subtriangularis Teiling & $\mathrm{F}$ & $\mathrm{R}$ & $\mathrm{R}$ \\
\hline Tetrastrum triangulare Komárek & $\mathrm{R}$ & $\mathrm{F}$ & $\mathrm{F}$ \\
\hline Westella botryoides De Wildeman & $\mathrm{F}$ & $\mathrm{S}$ & $\mathrm{R}$ \\
\hline \multicolumn{4}{|l|}{ Charophyta } \\
\hline Desmidium aptogonum Brébisson ex Kützing & $\mathrm{F}$ & $\mathrm{R}$ & $\mathrm{F}$ \\
\hline \multicolumn{4}{|l|}{ Cyanophyta } \\
\hline Chroococcus turgidus Nägeli & $\mathrm{s}$ & $\mathrm{s}$ & $\mathrm{F}$ \\
\hline Merismopedia punctata Meyen & $\mathrm{D}$ & $\mathrm{s}$ & $\mathrm{s}$ \\
\hline
\end{tabular}


Sustainability, Agri, Food and Environmental Research 4(1), 2016: 1-21

ISSN: 0719-3726

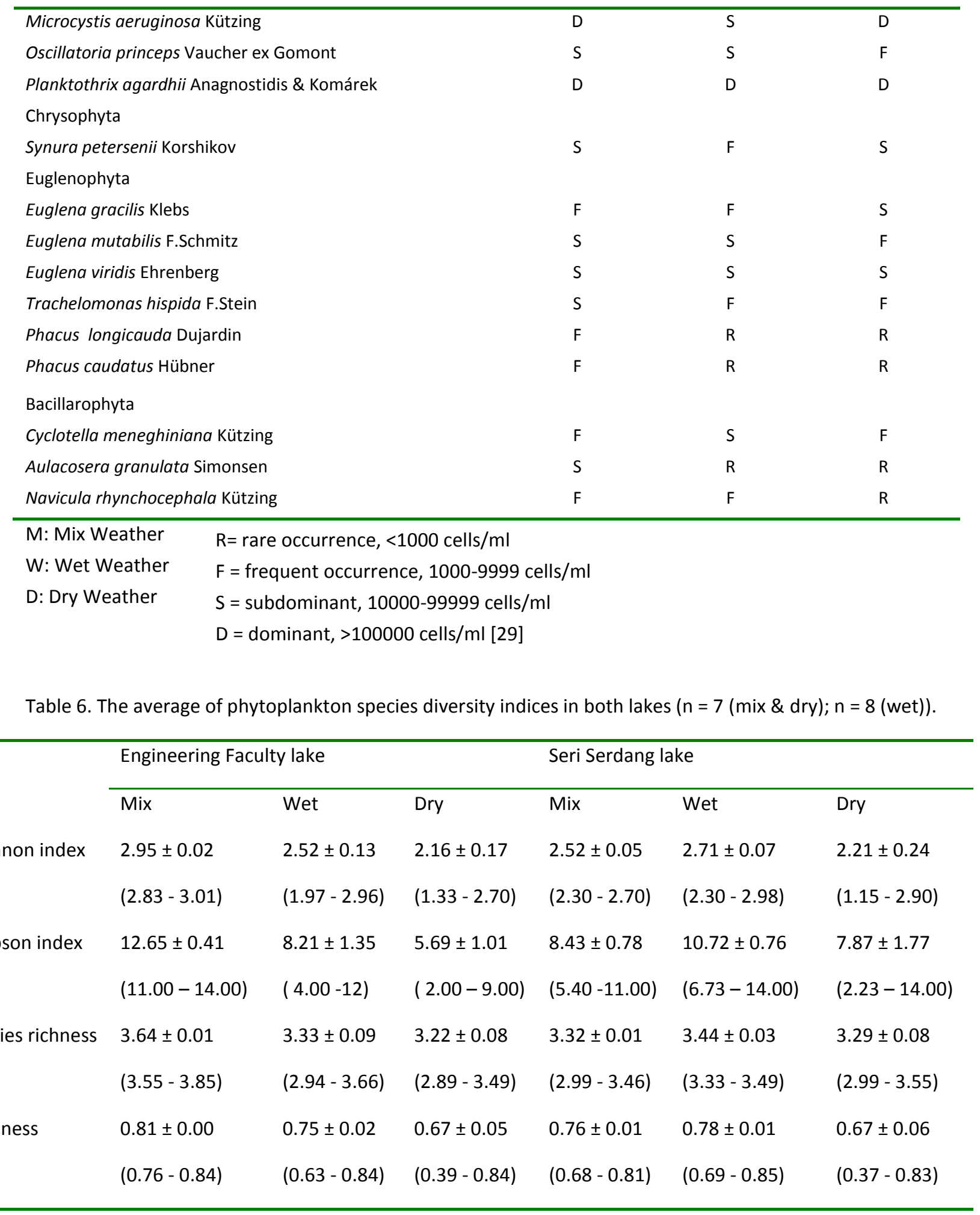


Sustainability, Agri, Food and Environmental Research 4(1), 2016: 1-21

ISSN: 0719-3726

Table 7. Robust species in Faculty of Engineering Lake and Seri Serdang Lake during different weather conditions.

\begin{tabular}{|c|c|c|c|c|c|c|c|c|}
\hline & Engineering faculty lake & & & & Seri Serdang lake & & & \\
\hline division & species & $\operatorname{mix}$ & wet & dry & species & $\operatorname{mix}$ & wet & dry \\
\hline \multirow[t]{18}{*}{ Chlorophyta } & Chlamydomonas reinhardtii & + & + & + & Chlamydomonas reinhardtii & + & + & + \\
\hline & Cosmarium depressum & + & - & - & kirchneriella obesa & + & + & - \\
\hline & Crucigenia crucifera & - & - & + & Monoraphidium griffithii & + & + & + \\
\hline & Crucigenia quadrata & + & - & - & Oocystis borgei & + & + & + \\
\hline & Kirchneriella obesa & + & - & - & Pandorina morum & + & - & + \\
\hline & Monoraphidium griffithii & + & - & - & Actinastrum hontzchii & - & + & + \\
\hline & Scenedesmus quadricauda & + & + & + & Chlorella vulgaris & - & + & + \\
\hline & Scenedesmus acuminatus & + & + & - & Chlorogonium elongatum & - & + & + \\
\hline & Scenedesmus bicaudatus & + & + & + & Golenkinia paucispina & - & + & + \\
\hline & Scenedesmus obliquus & + & + & + & Haematococcus pluvialis & - & + & + \\
\hline & Oocystis borgei & + & + & + & Micractinium pusillum & - & + & + \\
\hline & Pediastrum dúplex & - & + & - & Pteromonas aculeata & - & + & - \\
\hline & Scenedesmus ellipticus & - & + & - & Scenedesmus bicaudata & - & + & - \\
\hline & Dictyosphaerium pulchellum & - & - & + & Lagerheimia ciliate & - & - & + \\
\hline & Gonium pectorale & - & - & + & Scenedesmus dimorphus & - & - & + \\
\hline & scenedesmus abundans & - & - & + & & & & \\
\hline & Tetraderon minimum & - & - & + & & & & \\
\hline & Lagerhemia ciliate & - & - & + & & & & \\
\hline \multirow[t]{5}{*}{ Cyanophyta } & Chrococcus turgidus & - & + & - & Chrococcus turgidus & - & + & - \\
\hline & Microcystis aeruginosa & + & + & + & Merismopedia punctata & + & + & - \\
\hline & Microcysti wesenbergii & + & + & - & Microcystis aeruginosa & + & + & + \\
\hline & & & & & Oscilltoria limosa & + & - & - \\
\hline & & & & & Planktothrix agardhii & + & + & + \\
\hline
\end{tabular}


Sustainability, Agri, Food and Environmental Research 4(1), 2016: 1-21

ISSN: 0719-3726

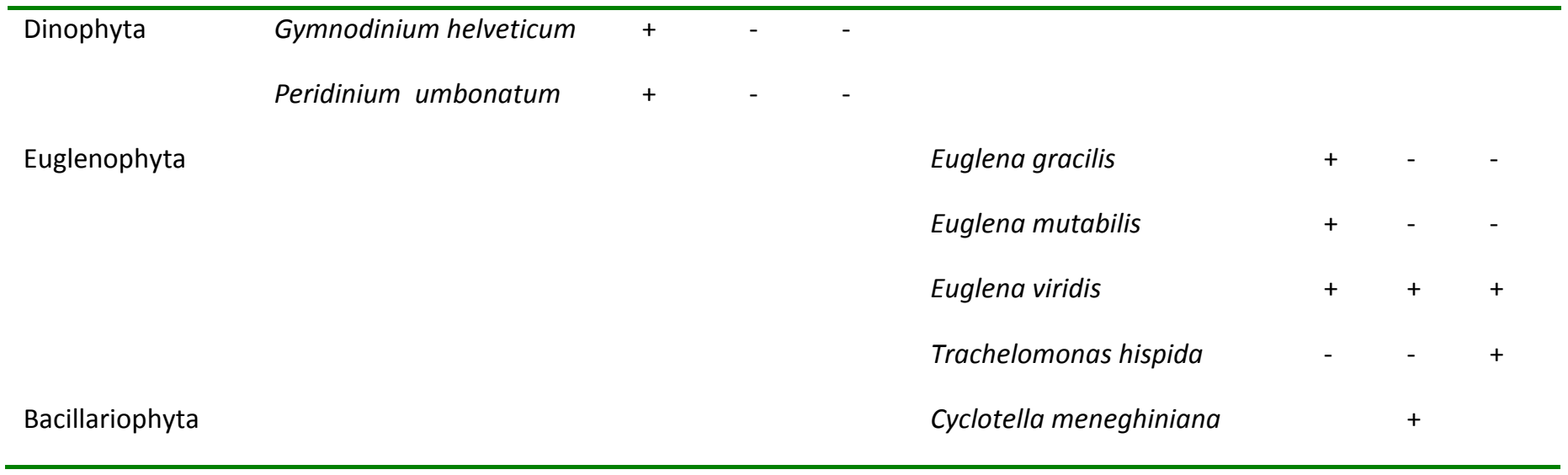

Table 8. Nygaard's Status indices for phytoplankton in the two lakes.

\begin{tabular}{|c|c|c|c|c|c|c|c|}
\hline \multirow{2}{*}{ Index } & \multirow{2}{*}{$\begin{array}{l}\text { Trophic Status Indices } \\
\text { Oligotrophic/ Eutrophic ( Nygaard, } \\
\text { 1949) }\end{array}$} & \multicolumn{3}{|c|}{$\begin{array}{c}\text { Faculty of Engineering } \\
\text { Lake }\end{array}$} & \multicolumn{3}{|c|}{ Seri Serdang Lake } \\
\hline & & Mix & Wet & Dry & Mix & Wet & Dry \\
\hline Myxophycean & $0.0-0.40,1-3.0$ & 0.70 & 1.00 & 1.00 & 1.66 & 2.50 & 5.00 \\
\hline Chlorophycean & $0.0-0.70,2-9.0$ & 5.00 & 7.20 & 6.20 & 10.33 & 15.50 & 31.00 \\
\hline Centric/Pennales & $0.0-0.30,0-1.75$ & 0.25 & 0.25 & 0.25 & 0.50 & 1.00 & 0.00 \\
\hline Euglenophycean & $0.0-0.70,0-1.0$ & 0.05 & 0.04 & 0.06 & 0.16 & 0.11 & 0.11 \\
\hline $\begin{array}{l}\text { Compound Quotient } \\
\text { (CQ) }\end{array}$ & $0.0-1.01,2-2.5$ & 6.10 & 8.80 & 7.80 & 14.30 & 20.50 & 41.00 \\
\hline
\end{tabular}




\section{CCA Map / Symmetric \\ (axes F1 and F2: $61.77 \%$ )}

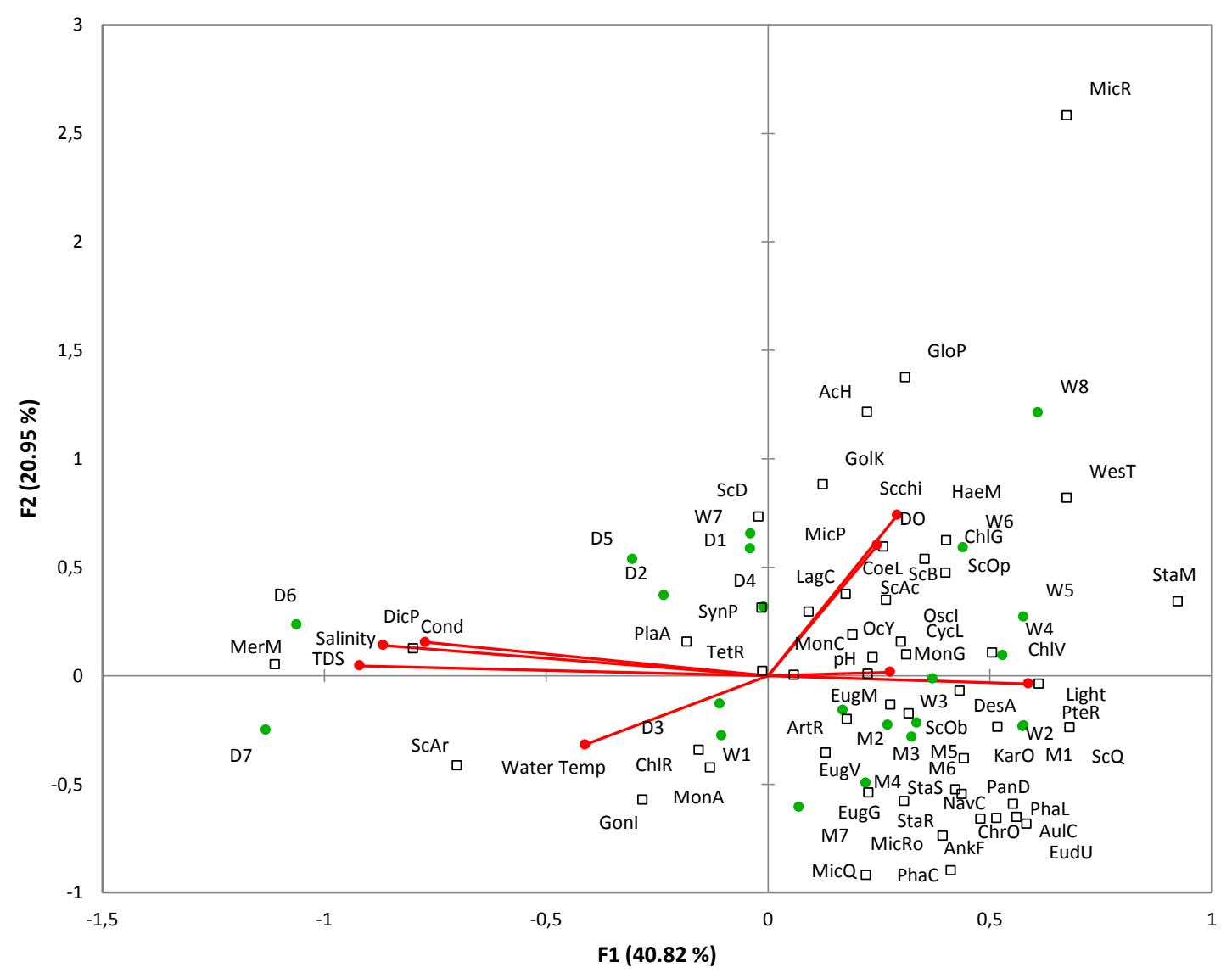

- Weather $\square$ Species • Variables

Fig. 4: CCA map for Seri Serdang Lake in different weather conditions. Abbreviations: Weather (W: Wet; D: Dry; M: Mix), Species (AcH: Actinastrum hontzchii; AnkF: Ankistrodesmus fusiformis; ChIR: Chlamydomonas reinhardatii; ChIV: Chlorella vulgaris; ChlG: Chlorogonium elongatum ; CoeL: Coelastrum microporum; ChrO: Chrococcus targidus DesA: Desmidium aptogonum; DicP: Dictyosphaerium pulchellum; EudU: Eudorina unicocca; EugG: Euglena gracilis; EugM: Euglena mutabilis; EugV: Euglena viridis ; GloP: Gloeocapsa punctate; GolK: Golenkinia radiata ; Gonl: Gonium pectorale; HaeM: Haematococcus pluvialis; KarO: Karchirenella obesa; LagC: Lagerheimia ciliate; MicQ: Micractinium quadrisetum; MicP: Micractinium pusillum; MicR: Micractinium radiate; MerM: Merismopedia punctate; MicRo: Microcystis urginosa; MonG: Monoraphidium griffithii; MonA: Monoraphidium arcuatum; MonC: Monoraphidium contortum; Oscl: Oscilltoria princeps; Ocy: Oocystis borgi; PanD: Pandorina morum; PhaL: Phacus longicauda; PhaC: Phacus caudatus; PlaA:Planktothrix agardhii; PteR:Pteromonas; ScQ:Scenedesmus quadricauda; ScAc: Scenedesmus acuminatus; ScAr: Scenedesmus armatus; ScOp: Scenedesmus opalensis; ScOb: Scenedesmus obliquus; ScB: Scenedesmus bicaudata; ScD: Scenedesmus dimorphis; StaR: Staurastrum; StaM: Staurastrum muticum; StaS: Staurodesmus subtriangularis; SynP: Synura petersenii; TetR: Tetrastrum triangulare; WesT: Westella botryoides). Variables (Cond: Conductivity; TDS: Total Dissolved Solid; scchi: Secchi depth; Temp: Temperature; DO: dissolved oxygen). 
CCA Map / Symmetric

(axes F1 and F2: $54.65 \%$ )

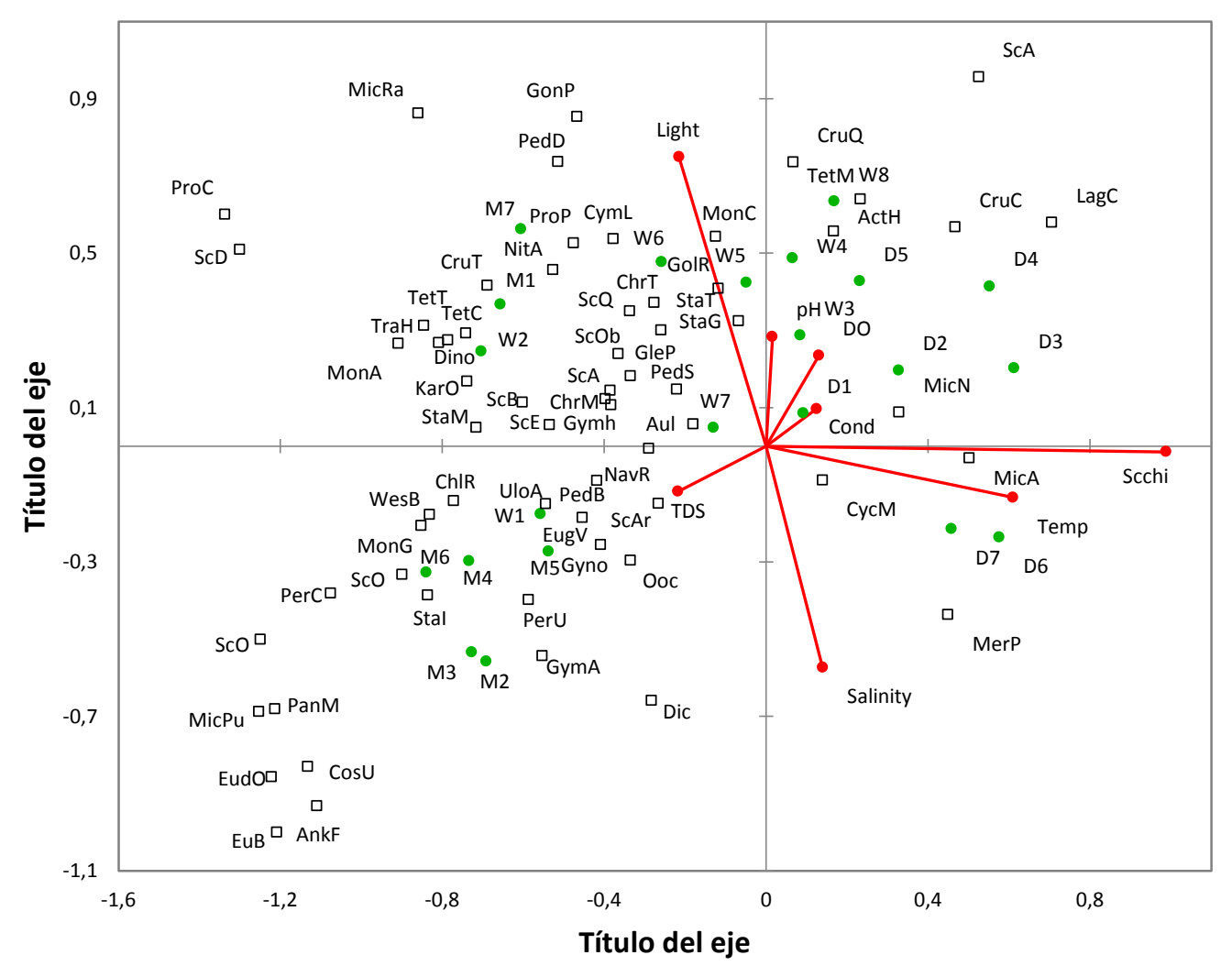

-Weather $\square$ Species • Variables

Fig. 5. CCA map for Engineering Faculty Lake in different weather conditions. Abbreviations: Weather (W: Wet; D: Dry; M: Mix), Species (ActH: Actinastrum hantzschii; AnkF: Ankistrodesmus fusiformis; Aul: Aulacoseira granulate; ChrM: Chrococcus minimus; ChrT: Chrococcus turgidus; ChIR: Chlamydomonas reinhardatii; CosU: Cosmarium undulatum; CruQ: Crucigenia quadrata; CruT: Crucigenia tetrapedia; CruC: Crucigenia crucifera; CycM: Cyclotella meneghinian; CymL: Cymbella lanceolate; Dic: Dictyosphaerium pulchellum ; Dino: Dinophysis acuta; EuB: Euastrum biverrucosum; EudO: Eudorina elegans; EugV: Euglena viridis; GolR: Golenkinia radiata; GonP: Gonium pectoral; Gymh:Gymnodinium helveticum; GymA: Gymnodinium aureolum; Gyno: Gyrodinium uncatenum; GleP: Glenodinium danicum; KarO: Karcherinella obesa; LagC: Lagerhemia ciliata; MerM: Merismopedia punctata; MicP: Micractinium pusillum; MicRa: Micractinium radiate; MicA: Microcystis aerurginosa; MicN: Microcystis wesenbergii; MonG: Monoraphidium griffithii; MonA: Monoraphidium arcuatum; MonC: Monoraphidium contortum; NavR: Navicula rhynchocephala; NitA: Nitzschia amphibian; Ooc:Oocystis borgei; PanM: Pandorina morum; PedB: Pediastrum boryainum; PedD: Pediastrum duplex; PedS: Pediastrum simplex; PerC: Peridinium cinctum; PerU: Peridinium umbonatum; ProC: prorocentrum micans; ProP: Protoperidinium thulesense;ScQ: Scenedesmus quadricauda; ScA: Scenedesmus acuminatus; ScAr: Scenedesmus armatus; ScB: Scenedesmus bicaudata; ScO: Scenedesmus obtusus; ScOP: Scenedesmus opalensis; ScOb: Scenedesmus obliquus; ScD: Scenedesmus dimorphus; ScA: Scenedesmus abundans; ScE: Scenedesmus ellipticus; StaM: Staurastrum muticum; StaT: Staurodesmus triangularis; Stal: Staurodesmus incus; StaG: Staurodesmus glaber; TetC: Tetraderon caudatum; TetM: Tetraderon minimum; TetT: Tetraderon triangularia; TraH: Trachelomonas hispida ;UloA: Ulothrix aequalis; WesB: Westella botryoides;).Variables (Cond: Conductivity; TDS: Total Dissolved Solid; scchi: Secchi depth; Temp: Temperature; DO: dissolved oxygen) 


\section{Discussion}

The data from CCA analysis suggested that the diversity of phytoplankton were influenced by type of lakes and weather conditions. Engineering lake which has low total dissolved solids, low conductivity, and low salinity is usually classified as oligotrophic lake. Seri Serdang as mesotrophic Lake is moderately low in water quality, moderately high nutrient, high conductivity, total dissolved solids, is favored by microalgae species. Wide distribution of phytoplankton species can be observed in Seri Serdang Lake during dry weather conditions when the salinity, total dissolved solids and conductivity were high.

Cloud cover during mix weather conditions influenced light penetration into the lake, therefor effect on photosynthesis and depression in dissolved oxygen in Water Lake, as could be observed from (Table 2) the average of dissolved oxygen was ( $\left.3.19 \mathrm{mg} \mathrm{L}^{-1}\right)$ and $\left(3.76 \mathrm{mg} \mathrm{L}^{-1}\right)$ in Engineering lake and Seri Serdang respectively. Rain fall during wet weather conditions influenced $\mathrm{pH}$ of lake water because of the acidic rain and reduced temperature. Furthermore, dilution impact of rainfall on total dissolved solids, conductivity, and salinity have been observed in both lakes during wet weather. During dry weather conditions water warmed up due to sunlight, led to increase in lake water temperature. Dry weather condition and high temperature increased evaporation thus, lowering water level, concentrating of present ions, and contributed to higher conductivity levels. This is why conductivity is influenced by water temperature, salinity and total dissolved solids. Decreasing in water flow as a result of low dilution factor of rain, steady input of nutrient from residential effluent can also contribute to conductivity through their impact on salinity.

From CCA analysis observation, during dry weather condition, species such as Merismopedia punctata, Dictyosphaerium pulchellum were with high cell densities when TDS, salinity and conductivity were high. Although temperature have not increased so much ( 2 degree only) but it was a controlling factor in dominant of Chlamydomonas reinhardtii, Scenedesmus armatus, Monoraphidium griffithii, and Gonium pectorale. $\mathrm{pH}$, secchi depth and dissolved oxygen were controlling factor during wet weather conditions.

Water quality has profound effect on the abundance and diversity of microalgae (Naqqiuddin et al., 2014). Chlorophytes was the most phytoplankton group encountered both in terms of abundance and frequency occurrence which was also dominant in many lakes in Malaysia like Putrajaya Lake (26\%) (Sorayya et al., 2011), and Chini Lake in Pahang (65\%) (Kutty, Ismail, Fong, 2001). Chlorophytes have the ability to live in wide range of nutrients and physical environments. Dominance of desmids to the low $\mathrm{pH}$, low conductivity and the oligotrophic nature of the water was mentioned in many phytoplankton studies, and similar finding attributed from the limnetic zone of Putrajaya Lake (Shariff, 2014), but in current study the desmids were not dominant. Additionally, desmids and diatoms are highly sensitive to environmental conditions change (Reynolds, 1998). Seasonal variation of chlorophytes showed that their density was high during mix weather conditions followed by wet weather conditions, but low during dry weather conditions due to the physiological effects of temperature and salinity (Martins, 1999). Pteromonas aculeata was more frequent during wet weather conditions and only in Seri Serdang Lake.

Dinophyta species was only appeared in Engineering Lake since they prefer oligotrophic lakes (Padisák et al., 1998). Dinoflagellates were found frequently during mix weather conditions and then the species quantity and the abundance of dinoflagellates dropped when the water temperature increased during dry weather conditions. Euglenophyta species was more frequent in Seri Serdang Lake as these species preferred more organic ecosystems condition and they indicate to the trophic states of freshwater lakes (Trifonova, 1998). Cyanophytes inhabit environments with diverse trophic states, at the surface of lake water or at the bottom (Mur, Skulberg, Utkilen, 1999). Cyanophyta which formed (11.5\%) in Seri Serdang lakes and (7.6\%) in Engineering lake have wide distribution in eutrophic lake. They used as a critical indicator for water quality because of their toxicity and their probable risks to human health. Growth of Cyanophytes increase with increasing temperature and organic contents in freshwaters bodies. This may explain why Merismopedia punctate, Microcystis aeruginosa, Oscilltoria princeps, and Planktothrix agardhii were dominant in Seri Serdang Lake during all-weather conditions. As the Engineering lake is categorized as an oligotrophic lake having low productivity and low organic contents, Microcystis aeruginosa, Microcystis wesenbergii and Merismopedia punctate were dominant during all weather conditions. Similar studies also found that Cyanophyta are the most common phytoplankton in Malaysian lakes, and they form (28\%) of phytoplankton in Putrajaya lake (Mansoor, 2012). There are only five species of Bacillarophyta found in 
engineering lake and three species in Seri Serdang Lake. Pennate diatoms were more frequent and they commonly occupy freshwater environments (Basavaraja, 2013). Cyclotella meneghiniana was dominant only during wet weather conditions and this species known as pollutant tolerance diatom. Chrysophytes which tolerate low light and low temperature recorded only by one species (Synura petersenii) in Seri Serdang Lake. Microcystis aeruginosa and Microcystis wesenbergii were dominant in Engineering Faculty Lake, although many studies reported that these species are common in mesotrophic and eutrophic lake (Rawson 1956, Reynolds, 1998). Pediastrum duplex which was frequent only during wet weather, can be found in wide range of trophic conditions from oligo-mesotrophic until eutrophic conditions (Prasertsin et al., 2014). Oligotrophic lakes characterized by a high number of phytoplankton species but have a small number of individuals, while mesotrophic lakes support small numbers of phytoplankton species, but large numbers of individuals for each species (Omar, 2010) where same finding were observed in current study. The lowest species diversity was observed in both lakes in dry weather conditions, while the highest was in mix weather conditions in Engineering Lake, and in wet weather conditions for Seri Serdang Lake. During wet weather conditions the species diversity indices decreased because of dilution factor and flooding due to heavy rains. On other hand, the low values of species diversity indices were recorded within dry weather conditions in the two lakes may be due to the effect of high surface water temperature on the physiological and biochemical process of some phytoplankton species. The wide ranges of values contributed with spatial and temporal variation of phytoplankton species indicate the heterogeneity of phytoplankton population in the two lakes. As shown in (Figure $2 \& 3$ ), cell density was high while species diversity was low during dry weather because certain species were only dominating. There is significant correlation coefficient between temperature $\left({ }^{\circ} \mathrm{C}\right)$ and Shannon Index at $(\mathrm{p}<0.05)$ level.

The trophic states indices for Myxophycean $(0.7-1)$ and chlorophycean $(5-7.20)$ and compound quotient $(6.1-8.8)$ at engineering Faculty Lake indicated to eutrophic conditions, while (centric / pennate) diatoms $(0.25)$ and Euglenophycean $(0.05-0.06)$ indicated to oligotrophic states of this lake. In contrast, all Nyggard's trophic states at Seri Serdang Lake indicated to eutrophic conditions, and the very high values of theirs confirmed the pollution stated for the Seri Serdang Lake.

According to the CCA, the lakes showed biological and limnological differences among the different weather conditions and weather is the main factor in phytoplankton variability and distributions. Lake Ecosystem affected mainly by weather therefore, phytoplankton dynamics changed during different weather conditions.

\section{Acknowledgements}

The author is grateful to Dr. Hishamuddin Omar for his comments and the revision of the manuscript.

\section{References}

Abdillah, N., \& Teo, F. Y. 2013. Water Quality Assessment of Sri Serdang Pond, Malaysia. Proceedings of IAHR World Congress.

Abdul Rahim Nik, Alexander K. Sayok, Efransjah and Sapuan Ahmad. 2007. Issues Affecting the Sustainability of Loagan Bunut Lake, Sarawak, and Paper presented at the Colloquium on Lakes and Reservoir Management: Status and Issues, 2 - 3 August, Putrajaya Malaysia.

Adeleke Abdul Rahman O., Nik Daud N.N., Ahsan Ammimul, Biswajeet Pradhan. 2014. Water Quality Assessment of UPM Lake and the Impact of Geographic Information System. International Journal of Environmental Monitoring and Analysis, 2: 158-162. doi: 10.11648/j.ijema.20140203.15 
Azril Mohamed Shaffril, H., Abu Samah, B., Lawrence d'silva, J., Sulaiman, \& Yassin, M. 2013. The process of social adaptation towards climate change among Malaysian fishermen. International Journal of Climate Change Strategies and Management, 5:38-53.http://dx.doi.org/10.1108/17568691311299354

Basavaraja, D., Narayana, J., Puttaiah, E. T., \& Prakash, K. 2013. Phytoplankton species diversity indices in Anjanapura reservoir, Western Ghat region, India. http://imsear.hellis.org/handle/123456789/148600

Bellinger, E. G., \& Sigee, D. C. 2010. Introduction to Freshwater Algae. Freshwater Algae: Identification and Use as Bioindicators, 1-40.

Corcoran, E. (Ed.). 2010. Sick water? The central role of wastewater management in sustainable development: a rapid response assessment. UNEP/Earth print.

Dubey, S. 2013. Acid Rain-The Major Cause of Pollution: Its Causes, Effects and Solution. International Journal of Scientific Engineering and Technology, 2: 772-775.

Edler, L., \& Elbrächter, M. 2010. The Utermöhl method for quantitative phytoplankton analysis. Microscopic and molecular methods for quantitative phytoplankton analysis, 110.

Goldstein, M. 2002. The complete idiot's guide to weather. Penguin.

Kumar, M., Naik, S. K., Murari, A., \& Rani, Y. 2012. Methods of diatom test: review of literatures. Journal of Forensic Medicine and Toxicology, 29: 47-54.

Kutty, A. A., Ismail, A., \& Fong, C. S. 2001. A preliminary study of phytoplankton at Lake Chini, Pahang. Pakistan Journal of Biological Sciences, 4: 309-313.

Mansoor Abdullah, H. 2012. A preliminary study on automated freshwater algae recognition and classification system (Doctoral dissertation, University of Malaya).

Margalef, D. R. 1958. Information theory in ecology. Society for General Systems Research.

Martins, I., Oliveira, J. M., Flindt, M. R., \& Marques, J. C. 1999. The effect of salinity on the growth rate of the macroalgae Enteromorpha intestinalis (Chlorophyta) in the Mondego estuary (west Portugal). Acta Oecologica, 20: 259-265. DOI: 10.1016/S1146-609X (99)00140-X.

Mccormick, M., Büntgen, U., Cane, M. A., Cook, E. R., Harper, K., Huybers, P. \& Tegel, W. 2012. Climate change during and after the Roman Empire: Reconstructing the past from scientific and historical evidence. Journal of Interdisciplinary History, 43: 169-220. doi/suppl/10.1162/JINH_a_00379.

Mir, S. I., Sahid, I., Gasim, M. B., Rahim, S. A., \& Toriman, M. E. 2015. Prediction of Soil and Nutrient Losses from the Lake Chini Watershed, Pahang, Malaysia. Journal of Physical Science, 26:53-70.

Mur, R., Skulberg, O. M., \& Utkilen, H. 1999. Cyanobacteria in the environment, In: Toxic Cyanobacteria in Water: A guide to their public health consequences, monitoring and management, Ingrid Chorus and Jamie Bartram.

Naqqiuddin, M. A., Alim, A., Ghani, I. A., Omar, H., \& Ismail A. 2013. The diversity and density of Marine dinoflagellates in the middle zone of the Straits of Malacca, The Malayan Nature Journal, 65: 61-80.

Naqqiuddin, M. A., Nor, N. M., Alim, A., Ghani, I. A., Omar, H., \& Ismail, A. 2014. Comparison between the Diversity and Density of Marine Dinoflagellates in Northern and Southern zone of Malacca straits. International Journal of Current Microbiology and Applied Sciences, 3: 723-748. 
Nõges, P., \& Tuvikene, L. 2012. Spatial and annual variability of environmental and phytoplankton indicators in Lake Võrtsjärv: implications for water quality monitoring. Estonian Journal Ecology, 61: 227-246. Doi: 10.3176/eco.2012.4.01

Nygaard, G. 1949. Hydrobiological studies on some Danish ponds and lakes. I kommission hos Ejnar Munksgaard.

Omar, S. Z., Shaffril, H. A. M., Kamaruddin, N., Bolong, J., \& D'silva, J. L. 2013. Weather Forecasting as an early warning system: Pattern of weather forecast usage among coastal communities in Malaysia. Life Science Journal, 10:2200-2215.

Omar, W. M. W. 2010. Perspectives on the use of algae as biological indicators for monitoring and protecting aquatic environments, with special reference to Malaysian freshwater ecosystems. Tropical life sciences research, 21: 51 .

Padisák, J., Krienitz, L., Scheffler, W., Koschel, R., Kristiansen, J., \& Grigorszky, I. 1998. Phytoplankton succession in the oligotrophic Lake Stechlin (Germany) in 1994 and 1995. In Phytoplankton and Trophic Gradients (pp. 179-197). Springer Netherlands.

Prasertsin, T., Pekkoh, J., Pathom-Aree, W., \& Peerapornpisal, Y. 2014. Diversity, New and Rare Taxa of Pediastrum spp. in Some Freshwater Resources in Thailand. Chiang Mai Journal of Science, 41: 1065-1076.

Rahman, H. A. 2009. Global climate change and its effects on human habitat and environment in Malaysia. Malaysian Journal of Environmental Management, 10: 17-32.

Ramaraj, R., TSAI, D. D. W., \& CHEN, P. H. 2014. Freshwater microalgae niche of air carbon dioxide mitigation. Ecological Engineering, 68: 47-52. DOI: 10.1016/j.ecoleng.2014.03.058

Rawson, D. S. 1956. Algal indicators of trophic lake types. Limnology and Oceanography, 1: 18-25. DOI: 10.4319/lo.1956.1.1.0018

Reynolds, C. S. 1998. What factors influence the species composition of phytoplankton in lakes of different trophic status? Hydrobiologia, 369:11-26. DOI: 10.1023/A: 1017062213207

Shannon, C. E., \& Weaver, W. 1949. The mathematical theory of information.

Shariff, M. 2014. Littoral and Limnetic Phytoplankton Distribution and Biodiversity in a Tropical Man-Made Lake, Malaysia. Advanced Studies in Biology, 6: 149-168. doi.org/10.12988/asb.2014.4631

Sharip, Z., \& Zakaria, S. 2007. Lakes and reservoir in Malaysia: Management and research challenges. In Proceedings of Taal2007: The 12th World Lake Conference 1349, 1355.

Sorayya, M., Aishah, S., Sapiyan, B. M., \& Mumtazah, S. S. 2011. A self-organizing map (SOM) guided rule based system for freshwater tropical algal analysis and prediction. Scientific Research and Essays, 6: 52795284.

DOI: $10.5897 /$ SRE11.866

Sørensen, T. 1948. A method of establishing groups of equal amplitude in plant sociology based on similarity of species and its application to analyses of the vegetation on Danish commons. Biologiske Skrifte, 5: 1-34.

Trifonova, I. S. 1998. Phytoplankton composition and biomass structure in relation to trophic gradient in some temperate and subarctic lakes of north-western Russia and the Prebaltic. Hydrobiologia, 369:99-108. Doi 10.1023/A: 1017074615932

Utermöhl, H. 1958. Toward the improvement of the quantitative phytoplankton method. MitteilungenInternationale Vereiningung fur Limnologie, 9: 1-38. Doi: 10.1234/12345678 
Vincent, W. F., Laurion, I., Pienitz, R., Anthony, K. M. W., \& Katey, M. 2012. Climate impacts on arctic lake ecosystems. Climatic Change and Global Warming of Inland Waters: Impacts and Mitigation for Ecosystems and Societies, 27-42.

Websites: Accuweather.com 
Sustainability, Agri, Food and Environmental Research 4(1), 2016: 1-21

ISSN: 0719-3726 